\title{
Search behavior of dogs (Canis familiaris) in invisible displacement problems
}

\author{
SYLVAIN GAGNON \\ Université du Québec à Montréal, Montréal, Québec, Canada \\ and \\ FRANÇOIS Y. DORÉ \\ Université Laval, Québec, Canada
}

\begin{abstract}
Gagnon and Dore (1992) showed that domestic dogs are able to solve a Piagetian object permanence task called the invisible displacement problem. A toy is hidden in a container which is moved behind a screen where the toy is removed and left. Dogs make more errors in these problems than they do in visible displacement tests, in which the object is hidden directly behind the target screen. In Experiment 1, we examined components of the standard procedure of invisible displacements that may make encoding or retention of the hiding location more difficult than it is in visible displacements. In Experiment 2, we compared dogs' performances in visible and invisible displacement problems when delays of 0,10 , and 20 sec were introduced between the object's final disappearance and the subject's release. The results revealed that dogs' poorer performance in invisible displacement tests is related to the complex sequence of events that have to be encoded or remembered as well as to a difficulty in representing the position change that is signaled, but not directly perceived.
\end{abstract}

For years, object permanence has been an important topic in the study of human cognitive development (Harris, 1975, 1983; Piaget, 1937/1967, Schubert, 1983). More recently, it has been integrated into the study of animal cognition (Antinucci, 1989; Doré \& Dumas, 1987; Étienne, 1973, 1984; Parker \& Gibson, 1990). Object permanence is defined by Piaget $(1937 / 1967)$ as the capacity to represent physical and social objects as distinct spatial elements of the environment. An organism that understands object permanence is aware that the objects in its environment are independent of its own actions and continue to exist when they cannot be directly perceived. Object permanence is essentially assessed through standardized visible and invisible displacement tests, in which subjects have to search for and find occluded objects (Décarie, 1965; Uzgiris \& Hunt, 1975). In both categories of tests, the standard test-

This paper reports portions of the research for a doctoral dissertation submitted by S.G. to the École de psychologie, Université Laval. It was supported by Grant A7030 from the Natural Sciences and Engineering Research Council of Canada (NSERC) and by Grant EQ 2760 from Fonds pour la Formation des Chercheurs et l'Aide à la Recherche (FCAR) du Gouvernement du Québec to F.Y.D., as well as by NSERC and FCAR scholarships to $S$.G. This research received approval from the Comite de protection des animaux de laboratoire de l'Université Laval, which is responsible for the application and enforcement of the rules of the Canadian Council on Animal Care. We thank Sandra DeBlois, Sylvain Fiset, Lucie Godbout, Danielle Lefebvre, and Martine Thibault for their assistance in collecting data. Special thanks are addressed to the breeders who have participated in this research, Ginette and Jacques Babin, Louyse and Richard Galibois, Jean-Louis Blais, Madeleine Charest, Martine Chabot, and finally, Sandra and Bob Larochelle. Correspondence should be addressed to S. Gagnon, Département de Psychologie, Université du Québec à Montréal, CP 8888, Succ. A, Montreal, Quebec, Canada H3C 3P8. ing procedure involves the use of a number of screens behind which an object that the subject will have to search for can be hidden.

In visible displacement tests, the object of search is moved, in full view of the subject, toward and behind the hiding screen. In human infants, understanding of these displacements develops during Stage 4 and Stage 5 of the sensorimotor period of intelligence (Piaget, 1937/1967). To succeed in these tests, the subject has to track the movements of the object and to search for it behind the last screen where he/she saw it disappear. In invisible displacement tests, the sequence of events is more complex and includes five distinct steps. First, the target object is moved inside a container. Second, the container holding the now hidden object is moved from its starting position to the back of the target screen. The third step is brief, but according to Piaget (1937/1967), it is the crucial event in invisible displacement problems. While the container is behind the target screen, the object is invisibly removed from the container and left in back of the screen. In the fourth step, the container is moved from behind the target screen and is rotated on its vertical axis to show the subject that it is now empty, thus giving an indirect cue that the object has been left behind the target screen. Using this cue, the subject can mentally reconstruct the invisible transfer of the object. In the final step, the container is returned to its final stationary position. In human infants, understanding of invisible displacements corresponds to Stage 6 of the sensorimotor period. To be successful, an organism has to understand two different things: (1) An object continues to exist even when it is not available to direct perception (a cognitive capacity that begins to develop in Stage 4), and (2) the location of an 
object can be predicted from its visible movements as well as from the mental reconstruction of movements that were not directly perceived but that were signaled.

Before claiming that a species can solve invisible displacement tests, one has to eliminate rival hypotheses that are more parsimonious. Natale, Spinozzi, Antinucci, and Poti (1986) have distinguished two kinds of solutions: one is representational and the other is not. Representational solutions refer to representation in the narrow sense given this term in the Piagetian literature. In invisible displacements, a solution is considered to be representational by Natale et al. if the subject finds the object by mentally reconstructing the position change from the container to the back of the hiding screen. A nonrepresentational solution involves a cue that the subject gradually learns to use (e.g., " pick the last screen touched by the experimenter") in order to retrieve the object. However, the nonrepresentational analysis is based on an assumption that can be questioned. If the term representation is used in its broader sense-that is, to refer to the memory of the object or the event-it is obvious that an organism must have some kind of representation to solve visible or invisible displacement problems because the organism is searching for an object that is no longer available to direct perception. Therefore, what Natale et al. (1986) call nonrepresentational is better off viewed as consisting of local rules acquired through practical apprenticeship but based nonetheless on a representation of the object's location.

Gagnon and Doré (1992) analyzed the possible strategies that dogs could use in solving invisible displacement tests and, in particular, different local rules that dogs could have learned. There was no sign of rule learning, and performance was significantly better than chance. Gagnon and Doré also demonstrated that olfactory cues are not effective in helping dogs to recover the target object. However, dogs were less successful in invisible displacement tests than they were in visible displacement problems.

In this paper, two questions about dogs' performances in invisible displacement tests were examined: First, why do dogs make more mistakes in invisible displacement tests than in visible displacement tests? Second, is the representation that dogs have of an invisible displacement problem as resistant to memory decay as is the representation they have of a visible displacement problem?

\section{EXPERIMENT 1}

Doré (1986, 1990; Goulet, Doré, \& Rousseau, 1993) showed that cats are unable to solve invisible displacement problems and examined two possible interpretations for this failure. It might result from an inability to specifically represent the invisible transfer of the object from the container to the target screen (Piagetian interpretation) or it might result from the complex sequence of events that have to be encoded or remembered.

Three factors related to encoding and retention were tested by Doré (1991; Goulet et al., 1992). One factor is the postdisappearance movement of the container
(Step 5) that may create retroactive interference with the memory of the object's hiding location. Another factor is the delay between the invisible transfer of the object and the subject's release (i.e., the time needed to bring the container back to its final position). The last factor is related to Step 2 of a standard invisible displacement problem. While concealed in the container, the object is moved from its starting position toward the target screen. Because the object is invisible for a short delay during this movement, the subject might have forgotten that the object was in the container. According to the Piagetian interpretation, this factor is not the key element in an invisible displacement test, but from an informationprocessing point of view, it makes sense that Step 2 might also negatively affect performance.

Doré (1991) tested the retroactive interference hypothesis in naive cats by including a postdisappearance movement in a visible displacement problem. He also added a condition which enabled him to control for the postdisappearance delay. Results confirmed that cats' performances were significantly disrupted by the movement of the container after the object had disappeared. However, this retroactive interference could not by itself explain the difference between visible and invisible displacement problems, because performances were still significantly lower in invisible displacement trials than in visible displacement trials with a postdisappearance movement of the container. Doré also found that a short postdisappearance delay had no significant effect on the performance in invisible displacement problems. These conclusions were confirmed by Goulet et al. (1993), who obtained similar results with experienced adult cats. In one of their studies, they also showed that adult cats are not influenced by the invisibility of the target object during the trajectory of the container from its starting position to the target screen. They concluded that cats failed invisible displacement problems because they are unable to mentally represent the invisible transfer of the target object from the container to the back of the screen (Step 3 of a standard invisible displacement problem).

As we have mentioned earlier, Gagnon and Doré (1992) have demonstrated that dogs displayed invisible-displacement performances that were significantly higher than expected by chance but significantly lower than performances in visible displacement problems. Therefore dogs, unlike cats, seem to be able to mentally reconstruct an invisible position change from an indirect cue. However, their level of success in invisible displacement problems could be lower than in visible displacement problems because of an encoding or retention deficit. One purpose of the following experiment was to test two of the three factors examined by Doré (1991; Goulet et al., 1993): retroactive interference from the postdisappearance movement of the container, and the invisibility of the object during its movement from starting position to the target screen. A procedure derived from Doré's experiments (Doré, 1991; Goulet et al., 1993) was used. Dogs' performances in standard visible and invisible displacement problems were 
compared with performances in visible displacement problems in which a postdisappearance movement of the container was added, and with performances in invisible displacement problems in which the object was visible during its movement from its starting position to the hiding screen.

\section{Method}

\section{Subjects}

Eleven purebred adult dogs ( 8 females and 3 males, mean age $=$ 2 years and 10 months, range $=1-6$ years) classified as sporting dogs (4 American cockers, 2 Gordon setters, 1 Labrador retriever) and terriers ( 4 Scottish terriers) by the American Kennel Club were recruited through breeders from the Quebec city area. Interbreed differences were not a relevant factor because Gagnon and Dore (1992) had already shown that dogs from different breeds exhibit comparable performances in object permanence tests. Seven of them had participated in previous experiments on object permanence, but there was at least 1 year between the last time they were tested and the present experiment. Testing was interrupted during 2 weeks for the American cockers because 2 females were in oestrus.

\section{Apparatus}

The screens that served to hide the target object were four white wooden boxes $(16.5 \mathrm{~cm}$ wide $\times 30 \mathrm{~cm}$ high $\times 12 \mathrm{~cm}$ deep) with a top and three sides but without a back panel (see Figure 1). They were fixed on a transparent plastic carpet $(176 \times 77 \mathrm{~cm})$. The bottom of each screen was reinforced with lead sheets in order to make them difficult for the subjects to displace. The screens were arrayed in a semicircle at a distance of $20 \mathrm{~cm}$ from each other, so that all were equidistant from the subject's starting position. Black stripes arrayed on the carpet indicated to the experimenter the position of each screen.

Squeezable rubber toys of different shapes and colors were used as target objects. Their height varied from 5 to $13 \mathrm{~cm}$. Many target objects were used within a testing session in order to maintain the subject's motivation. Each target object was fixed to the end of a $1-m$ invisible nylon thread. A rectangular container $(8 \times 18$

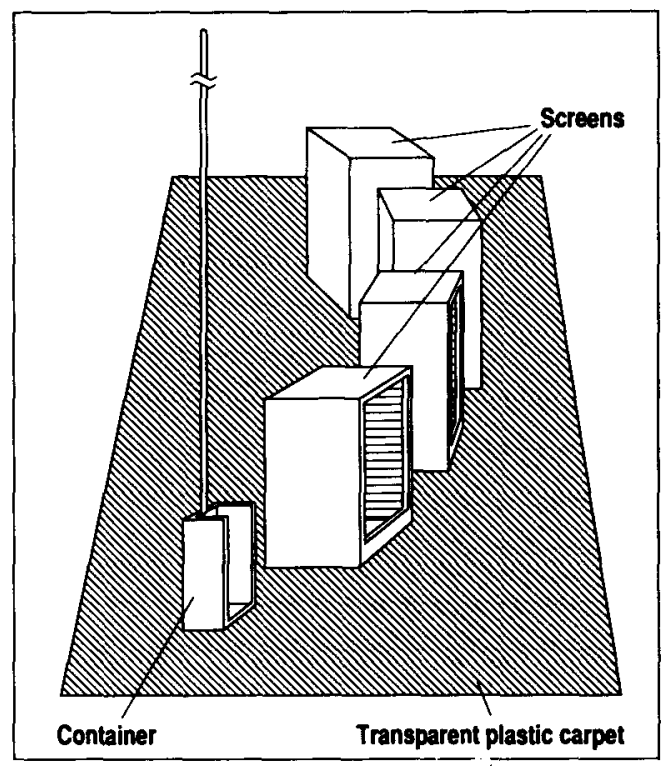

Figure 1. A schematic representation of the apparatus used in Experiments 1 and 2. $\times 8 \mathrm{~cm}$ ) made of wood and without a top or a back panel was used in the invisible displacement tests. This container was fixed to the extremity of a 1-m tubular plastic stick. It played the same role as the small cup or the experimenter's hand in infant testing of invisible displacements (i.e., the object was inside the container when it was moved). All sessions were videotaped.

\section{Procedure}

The subjects were tested in the owner's or breeder's home where a room was selected on the basis of three criteria: area (at least $4 \mathrm{~m}^{2}$ ), quality of illumination, and the dog's familiarity with this environment (the subject spent time daily in this room). The first session was aimed at shaping the basic response toward the target object. In a previous study (Gagnon \& Dore, 1992), dogs were not shaped because they showed a high interest in playing with the toys and were exposed to a relatively low number of trials. In this experiment, dogs were given several testing sessions, so a food reinforcement procedure was included to prevent a significant decrease of motivation during the experiment. The shaping criterion that dogs reached before being tested was 10 consecutive successful trials, defined as walking toward the target object and displaying one of the following responses: grasping the object with the mouth, touching it with the paw, or putting the muzzle right on the object. After each successful trial, the subjects were reinforced with bits of LAM dry commercial food, pieces of Nutriscience dog cookies, or bits of home cooked boiled liver. Every subject reached the criterion in one session.

During the 12 testing sessions, the animal was placed in front of the central screen at a distance of $1.5 \mathrm{~m}$ and was manually restrained by an experimenter who held its front shoulders, thus preventing the initiation of any movement toward the object or a particular screen. Another experimenter, who stood behind the central screens, manipulated the object with the nylon thread. Before each trial, this experimenter caught the subject's attention by pulling the nylon thread and thus moving the target object. As soon as the subject paid attention to the target object, the experimenter hid the object behind one of the screens as specified in the description of each test. When the object was totally hidden and the experimenter motionless, the subject was released by the other experimenter and allowed to search for the object. A trial was failed if the animal chose the wrong screen, looked behind the screen without displaying one of the three aforementioned responses, or did not make the appropriate choice and response in the minute that followed disappearance.

As we mentioned in a previous paper, there is a possibility of cuing, because the procedure involves a face-to-face interaction between the experimenter behind the screens and the subject. Obviously, this experimenter avoided as far as possible giving any cue to the subjects, and none of the dogs appeared (in videotape examinations of their behavior) to wait for reactions from the experimenter before or during their actions. In order to eliminate the possibility of cuing by the experimenter who held the subject, he/she kept his/her eyes closed during the displacement of the object and the postdisappearance delay. This control eliminated the possibility that the dogs could have been cued by this experimenter, because he/she did not know where the object was hidden.

The subjects were exposed to four different types of test: a standard visible displacement test (SV), a side-by-side visible displacement test (SSV), a standard invisible displacement test (SI), and a side-by-side invisible displacement test (SSI). Each testing session included 32 trials. Six testing sessions were composed of $16 \mathrm{SV}$ and 16 SI trials, whereas six sessions were composed of 16 SSV and 16 SSI trials. The trials within a session as well as the two kinds of sessions were randomly distributed.

Standard visible displacement test (SV). The container was placed at one or the other end of the semicircle formed by the four hiding screens (identified as A, B, C, and D, with Screen A being 
at the extreme left from the subject's viewpoint) and was not moved during the trial. In this test, the object was visibly moved toward, and hidden behind, one of the four screens. The target screen varied from trial to trial, and the object was hidden an equal number of times behind each of the four screens.

Side-by-side visible displacement (SSV). In this test, the experimenter brought the target object beside the container at its starting position and moved both the target object and the container side by side toward the target screen. Then, the target object was visibly hidden behind the target screen and the container was brought back to its final position. The starting and final positions of the container were either to the left of Screen A or to the right of Screen D. The target screen varied from trial to trial, and the object was hidden an equal number of times behind each screen.

Standard invisible displacement test (SI). In this test, the experimenter initially moved the target object into the container at its starting position, the open side facing the subject. The experimenter then rotated the container $180^{\circ}$ on its vertical axis, its open side now facing the experimenter, and moved it behind the target screen, where the object was invisibly removed from the container and left behind the screen. As soon as the container came out from behind the target screen, the experimenter again rotated the container $180^{\circ}$ on its vertical axis, its open side now facing the subject, in order to show the subject that the object was no longer in the container. Finally, the container was brought back to its final position. The starting and final positions of the container were the ends of the semicircle. As in SV and SSV trials, target screens varied from trial to trial and the object was hidden an equal number of times behind each screen.

Side-by-side invisible displacement test (SSI). In SSI trials, only the last three steps of the invisible displacement test were kept; in other words, subjects could see both the object and the container during their displacement to the target screen. The target object was first moved near the container at its starting position. Then, both the target object and the container were moved side by side close to the target screen. At this moment, the object was moved into the container (its open side facing the subject), the experimenter rotated the container $180^{\circ}$ on its vertical axis (its open side now facing the experimenter), and both the container and the object were moved behind the target screen. The object was then invisibly removed from the container and left behind the target screen. As soon as the container came out from behind the target screen, the experimenter again rotated the container $180^{\circ}$ on its vertical axis, its open side now facing the subject, in order to show to the subject that the object was no longer in the container. Finally, the container was brought back to its final position. The starting and final positions of the container were the ends of the semicircle. The target screen varied from trial to trial, and the object was hidden an equal number of times behind each screen.

In SI and SSI trials, the container was rotated on itself while it was brought to its final position, thus showing the subject that the object was no longer in the container. Natale et al. (1986) have asserted that the container used for invisible displacement should not be shown empty to the subject. According to them, an organism that understands invisible displacements should search for the object sequentially: first in the container, which is the first place where the object has disappeared, and then behind the target screen. As Gagnon and Dore (1992) have pointed out, sequential search is not the only way to discriminate between so-called representational solutions and local rules. Why should an organism necessarily make a sequential search? An organism can search directly for the object behind the target screen if it has made the required mental reconstruction of the invisible transfer when the empty container came out from behind the target screen.

In this experiment, we mixed both possible procedures. The container was shown empty (the open side facing the subject) to the subject after the object was left behind a screen, but in half of the sessions the experimenter again rotated the container $180^{\circ}$ on its vertical axis, its open side facing the experimenter. Trials in which the open side of the container faced the subject at the end of the manipulation were called open container trials, whereas trials in which the open side of the container faced the experimenter were called closed container trials. On closed container trials, the trial was scored as a failure and terminated without reward if the dog went to the container to look behind it before it went to the screen where the food was hidden. On open container trials, we could not readily tell whether or when the dog was looking at the open container, so we did not terminate such trials even if the dog went first to the open container.

At the end of this experiment, each subject had received a total of 384 trials, 96 trials for each test. The subjects were tested approximately two to three times a week during a 4- to 6-week period.

\section{Results and Discussion}

All 4,224 trials of Experiment 1 involved a search attempt behind one of the four screens. This result shows that subjects were highly motivated to search for the object.

The analysis will be divided into two sections. First, performances in the four tests will be compared. Second, we will compare the results across the six sessions containing each type of test in order to detect any sign of local rule learning. Videotapes of the experiment were viewed by two judges, and a coefficient of agreement [agreements/(agreements + disagreements) $\times 100$ ] on different components of the dogs' search behavior was computed. This coefficient of agreement was based on $25 \%$ of the trials. The coefficient of agreement between the judges on the discrimination between failed and successful trials was perfect, both judges agreeing on every trial.

\section{Percentages of Success}

The first analysis examined the influence of the orientation of the container on performances in invisible displacement trials. For this analysis, invisible displacement trials (SI and SSI trials) were grouped and compared according to the orientation of the container at the end of a trial (closed or open). Because the object never disappeared behind the container in the visible displacement trials, the orientation of the container was only tested for invisible displacement trials. A $t$ test $[t(10)=1.83$, n.s. $]$ showed that there was no significant difference between closed container trials $(M=53.6, S D=13.3)$ and open container trials $(M=58.5, S D=17.3)$. This analysis confirmed that the orientation of the container at the end of a trial (closed or open) did not affect the dogs' accuracy. In fact, the dogs paid little attention to the orientation of the container at the end of a trial. They displayed a behavior toward the container (smelled it, touched it) in less than $5.6 \%$ of the SI and SSI trials with the open container and in less than $6 \%$ of the SI and SSI trials with the closed container.

A second analysis examined the mean percentages of success in the four different tests collapsed over the orientation of the container at the end of the trial. The dogs' performances were more accurate in visible (SV and SSV) than in invisible displacement tests (SI and SSI) (see Table 1). In the visible displacement trials, performance was better on SV than on SSV trials, and in invisible displace- 
Table 1

Percentages of Success in Experiment 1

\begin{tabular}{lc}
\hline & $M \pm S D$ \\
\hline Visible displacement tests & \\
SV trials & $97.6 \pm 4.9$ \\
SSV trials & $79.0 \pm 9.5$ \\
Invisible displacement tests & \\
SI trials & $48.2 \pm 18.7$ \\
SSI trials & $64.2 \pm 12.0$ \\
\hline
\end{tabular}

ment trials, performance on SSI trials was better than on SI trials. A randomized-block analysis of variance (ANOVA) performed on these data showed that there was a significant difference between the four types of trials $[F(3,40)=32.05, p<.0001]$. Tukey's honestly significant difference (HSD) test $(p<.05)$ showed that SV and SSV trials were more successful than SI and SSI trials. This test also showed that there was a significant difference among visible displacement trials as well as among invisible displacement trials: SV trials were more successful than SSV trials and SSI trials were more successful than SI trials.

This analysis confirms our previous results (Gagnon \& Doré, 1992). Overall, the dogs' performance in visible displacement tests was significantly higher than in invisible displacement tests, and success in the invisible displacement tests was significantly higher than expected by chance [SI trials, $t(10)=4.00, p<.01$; SSI trials, $t(10)=8.45, p<.01]$.

The difference observed between SV and SSV trials suggests that the postdisappearance movement of the container retroactively interfered with dogs' memory of the target screen. In the visible displacement trials, the dogs' accuracy decreased from $97.6 \%$ in SV trials to $79.0 \%$ in SSV trials. The other factor that might contribute to poorer performance in the invisible displacement tests, the invisibility of the object while it is displaced from its starting position to the target screen, also seems to be relevant. In SI trials (the standard procedure), the object remained concealed in the container. In SSI trials, on the other hand, the dogs could visually track the object during its movement from its starting position to the target screen. Our results show that dogs had less difficulty recovering the target object when SSI procedure was used: Their performances increased from $48.2 \%$ in SI trials to $64.2 \%$ in SSI trials.

In summary, this analysis revealed three important results. First, it can be assumed that in invisible displacements, the final movement of the container creates some retroactive interference with dogs' memory of the hiding location because such interference also appeared in visible displacement trials with a postdisappearance movement of the container. Second, because performance was lower in SI than in SSI trials, the invisibility of the object during its movement toward the target screen is another factor that makes discrimination or retention of the hiding location more difficult in invisible than in visible displacements. However, a third factor that is related not to the complexity of the sequence of events, but rather to the nature of one of the events, also plays a crucial role. SSV and SSI trials were identical in terms of the visibility of the object during its movement toward the target screen as well as in terms of the container's final movement. Nevertheless, performance was poorer in SSI than in SSV trials. In SSI trials, the subjects could not see the object disappear behind the target screen, and they could not see the transfer of the object from the container to the target screen. Because the object was concealed in the container for only a fraction of a second before the container was moved behind the screen, the invisibility of the object as it is moved behind the target screen is probably not the main factor responsible for the difference of performance in SSV and SSI trials. Rather, the invisible transfer of the object from the container to the hiding screen is more likely to be the event that was difficult to represent.

\section{Local Rule Learning}

According to Gagnon and Dore (1992), there is no evidence that dogs solve invisible displacement problems by learning a local rule. If such learning had occurred, some improvement should be apparent across sessions.

Figure 2 shows the results of the six testing sessions. For each type of trial, the learning curve is pretty flat, although some small improvement in performance can be seen, especially on SSV and SSI trials. The improvement between Session 1 and Session 6 was $15 \%$ on SSV trials and $13 \%$ on SSI trials, while it was only $7 \%$ on both SV and SI trials. A trial type $\times$ sessions ANOVA showed that there was an overall difference between type of trial $[F(3,30)=79.27, p<.0001]$, as well as across sessions $[F(5,50)=3.77, p<.01]$. Although the interaction was not significant, separate randomized blocks ANOVAs performed for each type of test showed that there was a significant difference across sessions only for SSV trials $[F(5,50)=2.65, p<.05]$. Therefore, the latter analyses give no indication that dogs learned a local rule in SI and SSI trials.

Moreover, if we examine performances in the first testing session, the level of success in SI and SSI trials was already high: $t$ tests revealed that the level of success was

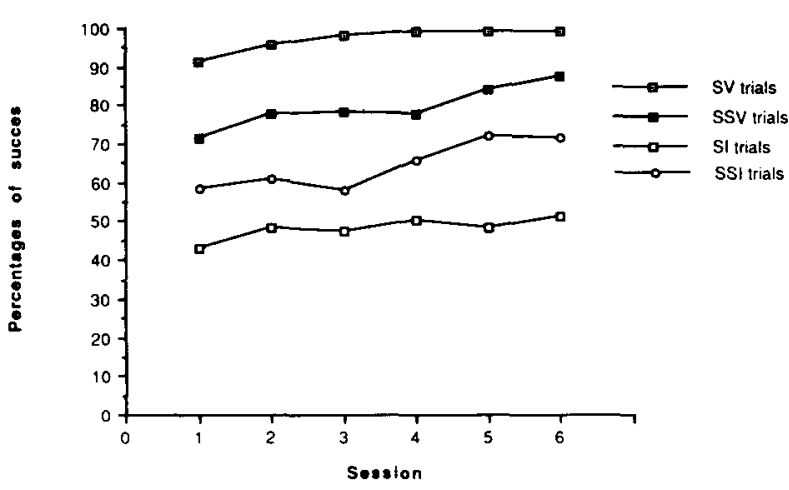

Figure 2. Percentages of success in each testing session of Experiment 1. 
higher than expected by chance $(25 \%)$ in both types of invisible displacement trials [SSI trials, $t(10)=7.51, p<$ .01 ; SI trials, $t(10)=3.52, p<.01]$. Thus, in the very first session, the subjects were able to find the object; they were not simply transformed from nonrecoverers to recoverers over sessions.

\section{EXPERIMENT 2}

The main purpose of Experiment 2 was to compare dogs' performances in both visible and invisible displacement problems when a delay was introduced between the disappearance of the target object behind the hiding screen and the dogs' release. If dogs' representation of invisible displacement problems is weaker than their representation of visible displacement problems, their performances should decrease more rapidly as a function of delay durations in invisible than in visible displacement problems. None of the research on object permanence conducted with nonhuman animals has tested the effect of a delay at the end of a trial in invisible displacement problems. However, effects of postdisappearance delays in visible displacement problems have been tested by Goulet and Doré (1989) and Fiset (1990) in domestic cats. They both found that at delays as short as $20 \mathrm{sec}$, cats' performance did not differ from chance. In this experiment, domestic dogs were tested at three different delays in both visible and invisible displacement problems: 0,10 , and $20 \mathrm{sec}$.

\section{Method \\ Subjects \\ Ten purebred adult dogs $(5$ males and 5 females, mean age $=$ 3 years and 5 months, range $=1-7$ years) classified as sporting dogs (4 American cockers, 3 Labrador retrievers) and terriers (3 Scottish terriers) were recruited in the Quebec city area. Five of the 10 subjects had participated in Experiment 1, but at least 3 weeks intervened between the two experiments. The other 5 subjects were experimentally naive. Testing for 1 subject (a Labrador retriever) had to be interrupted after Session 8 for a 2 -month period (the subject was out of town for dog shows), but was resumed thereafter.}

\section{Apparatus}

The apparatus was identical in every respect to that used in Experiment 1 . Labrador retrievers were tested with a tennis ball instead of the squeezable toys because they were more motivated by this target object.

\section{Procedure}

Each subject was first shaped to touch the object as in Experiment 1 . Nine subjects reached the criterion of 10 successful trials out of 10 in only 1 session, and 1 subject reached it in two sessions.

Each of the 12 testing sessions included 12 trials. The subjects were given two different tests-a standard visible displacement test (SV trials), and a standard invisible displacement test (SI trials). A session included six trials from each test.

Standard visible displacement trials (SV trials). The container was placed at one end of the semicircle formed by the four hiding screens, and did not move during the trial. In this test, the object was hidden behind one of the four screens and left there. The target screen varied from trial to trial, and the object was hidden an equal number of times under each of the four screens.
Standard invisible displacement trials (SI trials). The target object was moved into the container at its starting position, its open side facing the subject. The experimenter then rotated the container $180^{\circ}$ on its vertical axis, its open side now facing the experimenter, and moved it behind the target screen, where the object was invisibly removed from the container and left behind the screen. As soon as the container came out from behind the target screen, the experimenter rotated it again $180^{\circ}$ on its vertical axis, its open side now facing the subject, in order to show to the subject that the object was no longer in the container. Finally, the empty container was brought to its final position. The starting and final positions of the container were the ends of the semicircle. As in SV trials, the target screens varied from trial to trial and the object was hidden an equal number of times behind each screen.

In both SV and SI trials, there were three different postdisappearance delays, 0,10 , or $20 \mathrm{sec}$, defined as the interval that elapsed between the end of the manipulation and the subject's release. Because the final disappearance of the object behind the target screen in invisible displacement tests occurred approximately $3 \mathrm{sec}$ before the end of the manipulation (this was estimated as the maximal time necessary to bring the container to its final position), postdisappearance delays in visible displacement tests were 3,13 , and 23 sec. The intertrial interval was $30 \mathrm{sec}$. Of the 12 trials within a session, 6 were SV and 6 were SI trials, and each delay occurred four times per session. In each session, the two types of trial and the three delays were randomly distributed.

\section{Results and Discussion}

In the 1,440 trials that were administered, none was failed because a subject did not make a search attempt in the 60-sec time limit. This result confirmed that the subjects were highly motivated.

The analysis will be divided into two sections. First, performances in visible and invisible displacement trials will be compared as a function of the delays. Second, alternative strategies such as visual fixation and local rule learning will be examined. Videotapes were viewed by the same two judges as in Experiment 1 . The judges perfectly agreed on the distinction between successful and failed trials.

\section{Percentages of Success}

Table 2 presents the dogs' performances in visible and invisible displacement trials at 0,10 , and $20 \mathrm{sec}$. As previously found by Gagnon and Doré (1992) and in Experiment 1 , the dogs' performances were more accurate in visible than in invisible displacement trials, and did not seem to vary as a function of delay duration. A trial $x$ delay ANOVA showed that there was a significant difference between the two types of trials $[F(1,9)=451.14$, $p<.0001]$, SV trials being more successful than

Table 2

Percentages of Success in SV and SI Trials as a Function of Postdisappearance Delays in Experiment 2

\begin{tabular}{lccc}
\hline & \multicolumn{3}{c}{ Postdisappearance Delays } \\
\cline { 2 - 4 } Tests & $0 \mathrm{sec}$ & $10 \mathrm{sec}$ & $20 \mathrm{sec}$ \\
\multicolumn{1}{c}{$M \pm S D$} & $M \pm S D$ & $M \pm S D$ \\
\hline SI trials & $87.4 \pm 11.9$ & $85.8 \pm 8.8$ & $86.7 \pm 7.8$ \\
SI trials & $40.7 \pm 10.6$ & $38.7 \pm 8.6$ & $41.3 \pm 14.0$ \\
\hline
\end{tabular}


SI trials. The analysis also showed no significant effect of delay or of the interaction between trial type and delay. It seems that in visible and invisible problems, performances are resistant to postdisappearance delays as long as $20 \mathrm{sec}$. In other words, the difference in dogs' performances between visible and invisible displacement trials cannot be explained simply by less rapid memory loss on SV problems over the range of delays studied here.

Although the dogs' results did not vary as a function of delay, their performances were lower than what was found in Experiment 1, both in visible and in invisible displacement trials (cf. Table 1). Performance on both types of trials after the shortest delays was about $10 \%$ lower here than in Experiment 1 (SV and SI trials). Nonetheless, the $t$ tests clearly showed that the dogs' performances on invisible displacement trials were significantly higher than expected by chance $(25 \%)$ [delay = $0 \mathrm{sec}, t(9)=4.68, p<.01$; delay $=10 \mathrm{sec}, t(9)=$ $5.04, p<.01 ;$ delay $=20 \mathrm{sec}, t(9)=3.68, p<.01]$. The poorer performances after short delays might be explained by the random distribution of different delay durations. This phenomenon was observed in a study on object permanence in cats (Goulet \& Doré, 1989) as well as in experiments on pigeons' memory (Honig, 1987). Using a delayed conditional discrimination task, Honig found that the performance following a given memory interval is affected by memory interval distributions: memory at medium delays $(5 \mathrm{sec})$ was better in a distribution of short (1-sec) and medium (5-sec) delays than in a distribution of medium (5-sec) and long (10-sec) delays. Honig proposed two explanations. First, subjects in the shortmedium distribution might attend to the stimulus more closely and thus remember it better. Second, because the average delay of reinforcement was shorter in the shortmedium distribution, memorial processes may have been stronger than in the medium-long distribution. Either explanation might explain the dogs' poorer performance in both visible and invisible displacements of Experiment 2.

\section{Alternative Strategies}

Two alternative strategies, local rule learning and visual fixation, that could have been used by our subjects to solve the object permanence problems were examined. Local rule learning was assessed by comparing dogs' performances in visible and invisible displacement trials across the 12 testing sessions. In this analysis, results from the three postdisappearance delays were pooled because there were no differential effects across sessions due to delay. Figure 3 presents the results of SV and SI trials for each of the 12 testing sessions. Performances did not seem to change systematically across the 12 testing sessions. A trial $\times$ session ANOVA (the error term was partitioned) showed a significant difference between the two types of trials $[F(1,9)=200.26, p<.0001]$, but neither the session main effect nor the interaction was significant. Therefore, there is no evidence that dogs' success in invisible displacement tests results from the gradual learning of a local rule. Otherwise, after 144 trials, the performance

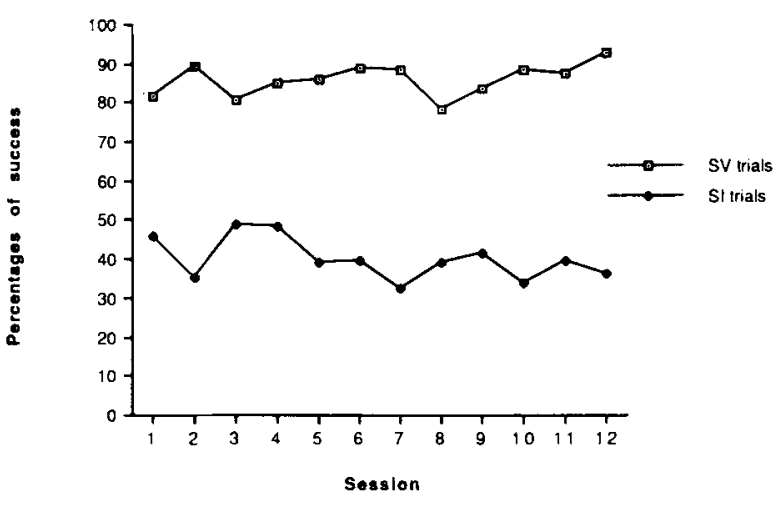

Figure 3. Percentages of success in each testing session of Experiment 2 .

on SI trials should have begun to approach performance on SV trials.

One might argue that dogs visually fixated the target screen during the entire delay. When both judges reviewed the videotapes of the experiment, they examined whether this was the case. Visual fixation was considered to be interrupted as soon as the subject turned its head away from the target screen (left or right, up or down). For delays of $10-$ and 20 -sec delays, the judges analyzed the visual fixation for the first half of the delay, and then for the second half. The coefficient of agreement between judges for the first half of the delays (which also included the 0 -sec delay trials) was $97.2 \%$, and for the second half of the 10 - and 20 -sec delays, it was $98.3 \%$. In short delays $(0 \mathrm{sec})$, the dogs interrupted their visual fixation on $85 \%$ of the visible displacement trials, and on $93 \%$ of the invisible displacement trials. With longer delays ( 10 and $20 \mathrm{sec}$ ), the visual fixation was practically absent, dogs interrupting it in $96.7 \%$ and in $99.2 \%$ of the visible and invisible displacement trials, respectively. Complete visual fixation was surely not necessary for our subjects to recover the target object in visible or invisible displacement trials.

In summary, the results from Experiment 2 confirmed that dogs' performances are more accurate in visible than in invisible displacement problems and revealed that their representation of the object's location, in both types of problem, is resistant to memory decay over $20 \mathrm{sec}$.

\section{GENERAL DISCUSSION}

In previous experiments, we showed that dogs' performances in invisible displacement problems, although higher than expected by chance, are significantly lower than in visible displacement problems. In Experiment 1, we found that the movement of the container at the end of an invisible displacement created some retroactive interference with the memory of the object's hiding location. Specifically, we observed that if the object's disappearance in visible displacement trials was followed by a movement of the container similar to the one that $\alpha c-$ 
curs in an invisible displacement problem, the dogs' performances were significantly lower than if not followed by this movement.

In Experiment 2, dogs were tested with short ( 0 - or 3$\mathrm{sec}$ ), medium (10- or 13-sec), and long (20- or 23-sec) postdisappearance delays, but their performance did not vary as a function of the delay duration either in visible or in invisible displacement trials. Thus, the dogs' capacity to find the object was resistant to memory decay over this range. On the other hand, as revealed by Experiment 1, the invisibility of the object during its movement toward the target screen is a relevant factor in the explanation of the dogs' poorer performance in invisible displacement trials. While the object was in the container, the subjects might have forgotten where it was and, therefore, had problems encoding its final hiding location.

Despite their relevance to the interpretation of dogs' search behavior, the invisibility of the object during its movement toward the target screen and any retroactive interference created by the container's final movement are not the only factors that affect dogs' performances. In fact, performances in visible and in invisible displacement trials still differ when these factors are controlled (comparison of SSV and SSI trials in Experiment 1). According to a Piagetian interpretation, this would mean that although dogs are successful in invisible displacement tests, their representation of a position change that is signaled but not directly perceived is not as good as their representation of events that are directly perceived. This may appear to be a truism, but the capacity to represent perceived movements versus movements that have to be inferred has not been systematically compared in studies of object permanence.

In domestic cats, these two cognitive capacities can be easily dissociated. Cats are able to predict the position of an object they see move and disappear, but they are unable to mentally reconstruct a movement (invisible transfer of the object from the container to the hiding screen) that is signaled (empty container) but not directly perceived (Doré, 1986, 1990; Goulet et al., 1993; Dumas \& Doré, 1989). Some species are able to solve invisible displacement problems, but the extent to which they are able to represent inferred movements is rarely assessed by a comparison with their capacity to represent perceived movements. There is convincing evidence, for example, that chimpanzees (Mathieu, Bouchard, Granger, \& Herscovitch, 1976; Wood, Moriarty, Gardner, \& Gardner, 1980) and gorillas (Natale et al., 1986; Redshaw, 1978) reach Stage 6 of object permanence and understand invisible displacements. There are indications that capuchin monkeys (Mathieu et al., 1976) and some psittacine birds (Pepperberg \& Funk, 1990; Pepperberg \& Kozak, 1986) are also able to infer and represent movements that they have not seen, although these results have been questioned (see Antinucci, 1989, and Gagnon \& Doré, 1992). Except for Mathieu et al.'s (1976) study, which compared performances of one chimpanzee in visible and in invisible displacement tests (one error out of 60 trials in invisible displacements and one error out of 120 in visible displacement tests), the other studies (including those with human infants) describe their subjects as being able to solve invisible displacement problems without further qualifications or systematic comparisons with their performance in visible displacement tests. Therefore, it is presently impossible to verify whether species other than dogs display Stage 6 search behavior without perfect understanding of inferred movements.

Studies of object permanence in animals have shown that there are interspecific differences in the kinds of visible displacements that animals can understand and represent (see Doré \& Dumas, 1987). Future research should investigate more thoroughly whether there are also differences among the species that solve invisible displacement problems in the incapacity to understand and represent movements that have to be inferred.

\section{REFERENCES}

Antinucci, F. (1989). The theoretical framework. In F. Antinucci (Ed.), Cognitive structure and development in nonhuman primates (pp. 11-17). Hillsdale, NJ: Erlbaum.

DECARIE, T. (1965). Intelligence and affectivity in early childhood. New York: International Universities Press.

DorE, F. Y. (1986). Object permanence in adult cats (Felis catus). Journal of Comparative Psychology, 100, 340-347.

DorE, F. Y. (1990). Search behaviour of cats (Felis catus) in an invisible displacement test: Cognition and experience. Canadian Journal of Psychology, 44, 359-370.

DorE, F. Y. (1991). Search behaviour of cats in an invisible displacement test: Object permanence and retroactive interference. In Cahiers de recherche de l'Ecole de Psychologie. Université Laval, Cité Universitaire, Quebec.

Doré, F. Y., \& Dumas, C. (1987). Psychology of animal cognition: Piagetian studies. Psychological Bulletin, 102, 219-233.

Dumas, C., \& DorE, F. Y. (1989). Cognitive development of kittens: A cross-sectional study of object permanence. Journal of Comparative Psychology, 103, 191-200.

ÉTIENNE, A. S. (1973). Searching behavior towards a disappearing prey in the domestic chick as affected by preliminary experience. Animal Behaviour, 21, 749-761.

Étienne, A. S. (1984). The meaning of object concept at different zoological levels. Human Development, 27, 309-320.

FiseT, S. (1990). Processus d'encodage de linformation spatiale par le chat domestique (Felis catus) [Spatial encoding in domestic cats]. Unpublished document, École de Psychologie, Université Laval, Cité Universitaire, Quebec.

GaGNON, S., \& DORE, F. Y. (1992). Search behavior in various breeds of adult dogs (Canis familiaris): Object permanence and olfactory cues. Journal of Comparative Psychology, 106, 58-68.

Goulet, S., \& DoRÉ, F. Y. (1989, July). Permanencia del objecto y interferencia proactiva de la duracion del intervallo de retention en los gatos domesticos (Felis catus) [Object permanence and proactive interference of delay duration in domestic cats]. Paper presented at the XXIl Congreso Interamericano de Psicologia, Buenos Aires.

Goulet, S., DORE, F. Y., \& Rousseau, R. (1993). Object permanence and working memory in cats. Manuscript submitted for publication.

Harris, P. L. (1975). Development of search behavior and object permanence during infancy. Psychological Bulletin, 82, 332-344.

HarRis, P. L. (1983). Infant cognition. In M. M. Haith \& J. J. Campos (Eds.), Handbook of child psychology: Vol. 2, Infancy and developmental psychobiology (4th ed., pp. 689-782). New York: Wiley.

HoNIG, W. K. (1987). Memory interval distribution effects in pigeons. Animal Learning \& Behavior, 15, 6-14.

Mathieu, M., Bouchard, M. A., Granger, L., \& Herscovitch, J. (1976). Piagetian object permanence in Cebus capucinus, Lagothrica flavicauda and Pan troglodytes. Animal Behaviour, 24, 585-588.

Natale, F., Antinucci, F., Spinozzi, G., Poti, P. (1986). Stage 6 
object concept in nonhuman primate cognition: A comparison between Gorilla (Gorilla gorilla gorilla) and Japanese macaque (Macaca fuscata). Journal of Comparative Psychology, 100, 335-339.

PARKer, S. T., \& GiBSON, K. R. (1990). "Language" and intelligence in monkeys and apes. Cambridge: Cambridge University Press.

Pepperberg, I. M., \& Funk, F. S. (1990). Object permanence in four species of psittacine birds: An African Grey parrot (Psittacus erithacus), an Illiger mini macaw (Ara maracana), a parakeet (Melopsittacus undulatus), and a cockatiel (Nymphicus hollandicus). Animal Learning \& Behavior, 18, 97-108.

PepPerberg, I. M., \& KozaK, F. A. (1986). Object permanence in the African Grey parrot (Psittacus erithacus). Animal Learning \& Behavior, 14, 322-330.

Piaget, J. (1967). La construction du réel chez l'enfant [The construction of reality in the child]. Neuchatel, Switzerland: Delachaux \& Niestlé. (Original work published 1937)
Redshaw, M. (1978). Cognitive development in human and gorilla infants. Journal of Human Evolution, 7, 133-143.

SCHUBERT, R. E. (1983). The infant's search for objects: Alternatives to Piaget's theory of development. In L. P. Lipsitt \& C. Rovee-Collier (Eds.), Advances in infancy research. (Vol. 2, pp. 137-184). Norwood, NJ: Ablex.

UzGIRIS, I. C., \& Hunt, J. (1975). Assessment in infancy: Ordinal scales of psychological development. Champaign, IL: University of Illinois Press.

Wood, S., Moriarty, K. M., Gardner, B. T., \& Gardner, R. A. (1980). Object permanence in child and chimpanzee. Animal Learning \& Behavior, 8, 3-9.

(Manuscript received April 27, 1992;

revision accepted for publication November 23, 1992.) 